Conclusion The ongoing programme continues to connect and engage with staff - mental health, wellbeing and resilience are part of everyone's conversations. Staff stay connected and reach out - different staff are able to engage with different activities. The offer continues to be collaborative, involving colleagues from spiritual care, fundraising, supportive care and $\mathrm{HR}$, which makes it more sustainable and relevant.

\section{P-188 BURNOUT IN THE UK CHILDREN'S HOSPICE WORKFORCE: FINDINGS FROM THE SWICH STUDY}

Andrew Papworth, Bryony Beresford, Lorna Fraser, Suzanne Mukherjee, Andre Bedendo, Jo Taylor. University of York, York, UK

\subsection{6/spcare-2021-Hospice.204}

In the UK, the children's hospice sector has become increasingly concerned about levels of work-related stress among its staff. This stems from increasing evidence that staff wellbeing is associated with the quality, cost and safety of patient care, and an acknowledgment of the important role of those working in children's hospices.

The SWiCH study, conducted by the Martin House Research Centre at the University of York, aims to increase understanding about the work-related stressors and rewards experienced by children's hospice care staff, and to identify the staff support systems and organisational practices that offer the most potential to enhance wellbeing at work. This paper will present preliminary findings from the study.

A national survey of UK children's hospice organisations and their care staff was administered between May and November 2020. A total of 583 staff from 32 children's hospices responded.

Initial analysis of the survey shows that 12 percent of respondents were suffering from moderate to severe burnout. Overall, respondents had an average burnout score (on the Copenhagen Burnout Inventory) of $32.5(n=583)$, which compares to an average score of 45.7 amongst a small sample (c.2.5\%) of NHS staff surveyed during the COVID-19 pandemic. UK children's hospice staff who responded to the survey had an average Job Satisfaction score of $6.9(n=414)$, and an average Core Engagement score of $7.5 \quad(n=415)$, the latter of which compares to the average of 7.0 for NHS staff (2020).

Further data analysis for the study will explore how these outcomes relate to the work-related stressors and rewards of working in a children's hospice, and what staff support systems and organisational practices have an impact on psychological wellbeing.

\section{P-189 'OFF TO WAR WHEN I DIDN'T SIGN UP!', SUPPORTING HOSPICE STAFF MENTAL HEALTH DURING A GLOBAL PANDEMIC}

Laura Shukla, Jenni Homewood. St Helena Hospice, Colchester, UK

10.1136/spcare-2021-Hospice.205

Wellbeing and Mental Health initiatives put in place during the pandemic.

The emotional and psychological pressures of working within hospice care are well documented, however, the addition of a global pandemic plunged hospice workforces into an unknown and unchartered way of working. Suddenly the switch from dealing with the everyday death and dying of patients, changed to having staff's own mortality, fear and anxiety thrust into reality. Unforeseen challenges within the workplace led to many staff experiencing emotions and anxieties that could not have been predicted.

The quick succession of events that changed everyday practice at the beginning of the pandemic gave rise to a number of initiatives implemented to support staff mental health and wellbeing. These have included:

- Wobble room - a designated safe place for staff to visit and relax in.

- Wobble room booklet for those working offsite.

- Gifts of meals and drinks from local companies.

- Mindfulness and relaxation.

- Self-help videos.

The number of Mental Health First Aiders and freedom to speak up guardians has been increased across the workforce. In addition to this, staff were given information and contact details for a multitude of both national and local support avenues such as the Samaritans, NHS, Here for you and MIND, enabling support virtually through websites, webinars, online training and apps. Within the hospice a focus on wellbeing was central to communications on the workplace intranet. This then resulted in the sign up of 15 new workplace health champions who will support ongoing activities throughout the year. The initiatives that have proved successful will continue to be offered and available to staff as we transition away from COVID-19 and maintain a focus on staff wellbeing within the organisation.

\section{P-190 DEVELOPING THE SKILLS OF HOSPICE STAFF TO PROVIDE CLINICAL SUPERVISION}

${ }^{1,2}$ Andy Ashley, ${ }^{1}$ Katherine Downey, 'Sarah Popplestone-Helm. ${ }^{1}$ St Richard's Hospice, Worcester, UK; ${ }^{2}$ Herefordshire and Worcestershire Health and Care NHS Trust, Worcestershire, UK

\subsection{6/spcare-2021-Hospice.206}

There is a strong national driver from the Care Quality Commission and the Nursing \& Midwifery Council around the provision of clinical supervision. This has long been recognised as vital in supporting staff in palliative care with the emotional labour of work (Goodrich, Harrison, Cornwell, et al., 2015) and helps develop a learning culture which improves patient safety and care (Francis, 2013). During the pandemic, where high levels of staff stress (Pastrana, De Lima, Pettus, et al., 2021) have led to moral injury and burnout, clinical supervision is all the more important.

With the development of a supervision policy, we recognised certain groups of hospice staff were not accessing clinical supervision regularly and it was mainly provided externally. The project, led by the education department, set out to utilise the skills and experience of existing staff to create additional supervisors and began by running lunchtime workshops to engage interest. Over half the 230 staff at the hospice were clinicians, of which 15 attended two days of training led by the head of family support and consultant clinical psychologist. It included theory, practice, 